\title{
Generalised viscoelastic fibre at small strain
}

\author{
Kostas P. Soldatos
}

Received: 23 August 2020 / Accepted: 22 December 2020 / Published online: 3 March 2021

(C) The Author(s) 2021

\begin{abstract}
A straight elastic fibre is usually perceived as a one-dimensional structural component, and its similarity with a cylindrical rod makes its concept analogous, if not equivalent with the concept of an elastic spring. This analogy enables this communication to match the one-dimensional response of a relevant viscoelastic fibre with that of a viscoelastic spring and, hence, to describe its one-dimensional behaviour in the light of a new, generalised viscoelastic spring model. The model shares simultaneously properties of an elastic spring and an inelastic damper (dashpot) and this communication is interested on its applicability at small strain only. However, the form of its constitutive equation, which is based on the combined action of an internal energy function and a viscous flow potential, is non-linear as well as differential and, also, implicit in the stress. The model enables a posteriori determination of (i) the manner that the elastic and the inelastic parts of the fibre strain are assembled and form the observed total deformation, (ii) the part of stress that creates recoverable work and the part of stress wasted in energy dissipation, and (iii) the amount of work stored in the material as well as the amount of energy dissipation during the fibre deformation. A detailed analysis is presented for the case that small-strain, steady viscoelastic deformation takes place in a spatially homogeneous manner. This includes a complete relevant solution of the problem of interest and is accompanied by an adequate set of corresponding qualitative numerical results.
\end{abstract}

Keywords Constitutive equations - Small-strain viscoelastic deformations · Viscoelasticity · Viscoelastic fibre . Viscoelastic rod · Viscoelastic spring

\section{Introduction}

It is well known that the theory of fibre-reinforced materials was initiated [1,2] with the purpose to model behaviour of rubber-like and structural solids reinforced by very strong fibres (see also $[3,4]$ and early references therein). Later theoretical developments still serve that purpose and include the extension of the theory towards modelling the behaviour of fibre-reinforced fluids [5] as well as the behaviour of solid and fluid composites reinforced by strong fibres resistant in bending, stretching and twist (see [6-11] and references therein for more recent developments).

Over the last couple of decades, the theory is also applied successfully in modelling the behaviour of fibrereinforced biological materials. In this context, the early efforts of modelling soft, tube-like fibre-reinforced biolog-

K. P. Soldatos $(\bowtie)$

School of Mathematical Sciences, University of Nottingham, Nottingham NG7 2RD, UK

e-mail: kostas.soldatos@nottingham.ac.uk 
Fig. 1 The known analogy between a linearly elastic spring of elastic modulus $K$, and $\mathbf{b}$ a linearly elastic cylindrical rod of Young's modulus $E$, cross-sectional area $A$, and length $L ; f, \sigma$ and $e$ represent force, axial stress and elastic strain, respectively (a)

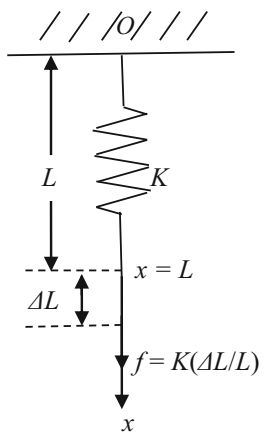

(b)

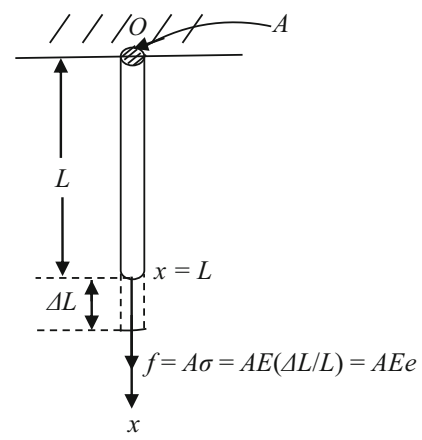

ical tissue $[12,13]$ were followed by substantial relevant progress, and also led to identification of fibre response that diverges considerably from that of the strong fibres considered in [1-11]. The existence and behavioural modelling of biological fibres that resist extension but do not support compression is thus already attracting considerable attention (see [14-16] and references therein). The same though does not seem to be true in the case that soft fibres exhibit viscoelastic behaviour (e.g. [17-19]). This communication thus aims to initiate a relevant modelling endeavour.

It is initially recalled that a straight elastic fibre is usually perceived as having the form of a flexible cord (e.g. $[1,2]$ ) or that of a slender cylindrical rod (e.g. [8]), or even the form of an Euler-Bernoulli beam in cases that the fibre can resist bending (e.g. [6]). In all cases though, it is macroscopically regarded as some kind of a onedimensional structure and, in this regard, its similarity with a straight cylindrical rod makes its concept analogous, if not equivalent to that of an elastic spring (e.g. [20,21] and Fig. 1). That analogy enables this communication to connect the one-dimensional response of a relevant viscoelastic fibre with that of the generalised viscoelastic spring model presented in [21]. It also enables adoption of a simplicity kind of notion employed in [21], according which the term "spring" is considered interchangeable/replaceable with the term "rod" and, hence, "fibre" in what follows, without further consequences. This notion is in fact unquestionable in elasticity, at least within the small strain/deformation regime (Fig. 1) which will become the range of applicability of the present analysis.

It is then further recalled that the generalised viscoelastic spring/rod/fibre model presented in [21] shares simultaneously properties of an elastic spring and an inelastic damper (dashpot). At its most general form, that model describes the behaviour of one-dimensional deformable solids subjected to large viscoelastic deformation but, through a series of progressively simplifying considerations, it produced a simpler relevant model that is applicable at small strain and deformation. That small-strain viscoelastic model is a very appropriate, essentially ideal point of departure for the more general research endeavour mentioned above, and thus becomes the focal point of this communication.

Under these considerations, and with the help of an Appendix, Sect. 2 presents the small-strain viscoelastic spring/fibre model in a manner that enables it to stand by itself, independently of its large deformation counterpart(s) developed in [21]. Nevertheless, the model is still underpinned by the combined action of a viscous flow potential that controls the inelastic part of its response and an internal energy function that dictates the manner that energy is stored in the material due to the elastic part of its total deformation. Although the model is considered applicable at small strain only, its constitutive equation still obtains a non-linear form, which is also differential as well as implicit in the stress, regardless of the form of either of the involved potentials. However, influenced by linear elasticity considerations, Sect. 3 combines next a quadratic form of the internal energy function with a quadratic viscous flow potential and, apart from presenting the relevant form obtained by the constitutive equation, it also discusses some fundamental energy and dissipation matters and describes in detail the manner that the elastic and the inelastic parts of the spring/fibre deformation are determined.

Section 4 achieves further analytical simplification by confining attention to the type of homogeneous deformation underpinned by the solution of the corresponding linearly elastic spring/fibre problem. In this manner, it introduces 
in a rational manner the case of steady or strictly quasi-static viscoelastic spring/fibre deformation. It is noted in passing that that point may alternatively be reached, independently of the modelling route followed in Sects. 2 and 3 , through a relevant linearised procedure followed and detailed in [21]. With the help of a second Appendix, Sect. 4 then presents the exact solution of the considered linear problem in the form of a fast converging series. Appropriate use of that series solution thus underpins the presentation of a set of qualitative numerical results that reveal the manner that the total deformation splits into elastic and inelastic parts.

Section 5 continuous the analysis and demonstrates the manner that (i) a part of the created energy is stored in the material while another part is dissipated, and (ii) the total stress acting on the spring/fibre splits into work-recoverable and energy-dissipative parts. Discussions of the outlined analytical results and corresponding computational progress continue into Sect. 6, which also highlights some important research steps and directions that may follow within well-known relevant research areas.

\section{The generalised viscoelastic spring/rod/fibre at small strain}

\subsection{Preliminaries: the linearly elastic spring/fibre}

The linearly elastic spring and rod/fibre depicted in Fig. 1 are considered equivalent one-dimensional elastic components, in the sense that they have the same length, $L$, they are both clamped at one end and, when the same external force of magnitude $f$ is applied at their free end, they undergo identical one-dimensional deformation. The spring has elastic modulus $K$ while its cylindrical rod/fibre equivalent has Young's modulus $E$ and cross-sectional area $A$. Through this well-known equivalence which, with the help of a single co-ordinate parameter, $x$, is adequately explained in the figure, these two linearly elastic models and associated terminology are considered completely interchangeable in what follows, without further notice or consequence.

It is accordingly assumed that, at time $t=t_{0}$, the spring/fibre is unloaded and unstressed at length $L$. Loading from that initial configuration takes off through application of a force,

$f(t)=T(t) A, \quad f\left(t_{0}\right)=T\left(t_{0}\right)=0$,

and, for $t>t_{0}$, gives rise to small strain

$e=u_{, x}$,

where $u(x, t)$ represents the displacement and a comma denotes partial differentiation with respect to $x$. As is further well-known, the strain energy stored internally in the elastically deforming spring,

$W^{e}(e)=\frac{1}{2} E e^{2} \geq 0$,

is quadratic in the strain and positive semi-definite; the equality sign applies only in the case of zero strain.

The quadratic form of this strain energy function thus gives rise to the linear constitutive equation

$\sigma=\frac{\partial W^{e}}{\partial e}=E e$

where $\sigma$ is the stress. Moreover, equilibrium requires

$\sigma_{, x}=\rho \dot{v}$ 
where a dot denotes differentiation with respect to time, $v=\dot{u}$ represents the velocity and $\rho$ is the material density. It is recalled, for later use, that changes of material density are generally considered negligible in linear elasticity and, at this point, $\rho$ is accordingly considered identical to its initial value, $\rho_{0}$, attained at $t=t_{0}$.

It is pointed out that Fig. 1 indicates that the boundary traction, $T(t)$, is tensile and, therefore, positive. For convenience, and unless is stated otherwise, this notion of tensile and positive $T(t)$ will be considered valid everywhere in what follows. It is left understood though that $T(t)$ may also be considered compressive, by appropriately reversing the sign of the affected analytical results, wherever this is necessary.

\subsection{The generalised, small-strain viscoelastic spring/fibre}

It is now postulated that the implied one-dimensional deformation comprises simultaneous action and/or interaction of elastic and inelastic deformation, the later being due to viscous fluid-type flow. The manner that those different parts of material response are merged and compose the observed total strain, $e$, is initially considered unknown.

It is, instead, anticipated that, due to inevitable energy dissipation, the elastic energy, $W$, stored internally in the viscoelastically deforming spring changes with, and therefore depends not only on the total strain, $e$, but also on the rate of the inelastic strain, $\dot{e}^{v}$, namely

$W=W\left(e, \dot{e}^{v}\right) \geq 0, \quad W(0,0)=0$,

where $\dot{e}^{v} \neq \dot{e}=\dot{u}_{, x}=v_{, x}$.

Moreover, such a dismissal of the purely elastic nature of the spring implies that potential changes of its material density may not anymore be negligible, even within the small-strain regime. The mass density evolution of the implied small-strain viscoelastic spring is thus required to also obey the standard continuity equation

$\dot{\rho}+\rho v_{, x}=0,\left.\quad \rho\right|_{t=t_{0}}=\rho_{0}$.

It is emphasised that the total strain involved in (2.6) is still defined in (2.2) but is not anymore elastic. On the other hand, the appearing rate of inelastic strain is defined as follows:

$\dot{e}^{v}=\frac{\mathrm{d} \phi(\sigma)}{\mathrm{d} \sigma}=\phi^{\prime}(\sigma)$,

where a prime denotes ordinary differentiation with respect to $\sigma$, and, as is shown in [21], the existence of a viscous flow potential $\phi(\sigma)$ guarantees that the rate of the dissipative work,

$\psi=\frac{1}{2} \sigma_{D} \dot{e}^{v}$,

is stationary.

Here, the stress is decomposed into two parts as follows:

$\sigma=\sigma_{R}+\sigma_{D}$

where $\sigma_{R}$ is the part that produces recoverable/elastic work while $\sigma_{D}$ is wasted in creation of dissipative work with rate $\psi$. Nevertheless, (2.5) still serves as the spring equation of motion (see also [21] as well as Appendix A).

The most general form of a constitutive equation associated with the outlined small-strain viscoelastic model is as follows:

$\sigma=\frac{\rho}{\rho_{0}}\left(\frac{\partial W}{\partial e}+\phi^{\prime \prime} \frac{\dot{\sigma}}{\dot{e}} \frac{\partial W}{\partial \dot{e}^{v}}\right)$ 
This is obtained in [21] through appropriate reduction of its large deformation counterpart into the small-strain deformation regime. As is though shown in Appendix A for self-sufficiency of this communication, it is also alternatively obtainable through direct use of appropriate small-deformation postulates/considerations. Despite its small-strain regime of applicability, this is generally a non-linear differential equation for $\sigma$, even in cases that its solution might be sought and found independently of the solution of the equilibrium and the continuity equations (2.5) and (2.7a), respectively.

In summary, the small-strain viscoelastic spring/rod/fibre model can take the form of the following onedimensional initial/boundary value problem: given the form of both the strain energy function (2.6) and the flow potential introduced in (2.8), determine the three principal unknowns, $u, \rho$ and $\sigma$, in a manner that satisfies the three principal differential equations, (2.5), (2.7a) and (2.11), subject to the boundary conditions

$\left.u\right|_{x=0}=0,\left.\quad \sigma\right|_{x=L}=T(t)$,

and the initial conditions (2.7b) and

$\left.u\right|_{t=t_{0}}=\left.\sigma\right|_{t=t_{0}}=0$.

After the implied principal unknowns thus are determined, and (2.2) is used for the calculation of the total strain $e$, the inelastic and elastic parts of the latter, as well as the elastic part of the displacement, can, respectively, be obtained as follows:

$e^{v}=\int_{t_{0}}^{t} \dot{e}^{v} \mathrm{~d} t=\int_{t_{0}}^{t} \phi^{\prime}(\sigma) \mathrm{d} t, \quad e^{e}=e-\int_{t_{0}}^{t} \phi^{\prime}(\sigma) \mathrm{d} t, \quad u^{e}=\int_{0}^{x} e^{e} \mathrm{~d} x=\int_{0}^{x}\left[e-\int_{t_{0}}^{t} \phi^{\prime}(\sigma) \mathrm{d} t\right] \mathrm{d} x$.

\section{Quadratic potentials and relevant consequences of the energy dissipation}

\subsection{Quadratic potentials}

It is natural for someone to search for simpler, possibly linearised forms of (2.11) by reducing as much as possible the influence that viscous flow exerts on the strain energy function, $W$. Influenced by the quadratic form that strain energy functions, such as (2.3), meet in linear elasticity, the strain energy function, $W$, as well as the viscous potential, $\phi(\sigma)$, are now both considered quadratic in their arguments.

It thus is initially assumed that

$W\left(e, \dot{e}^{v}\right)=\frac{1}{2} E e^{2}+\eta e \dot{e}^{v} \geq 0$,

where $\eta$ is an appropriate, viscosity related material modulus. An additional quadratic term that might involve $\left(\dot{e}^{v}\right)^{2}$ is not included in (3.1), as this would represent purely viscous material flow that is assumed of negligible influence on the stored internal energy.

The form (3.1) of $W$ clearly satisfies the initial condition (2.6b) and enables the constitutive equation (2.11) to acquire the more specific form

$\sigma=\frac{\rho}{\rho_{0}}\left\{\left(E+\eta \phi^{\prime \prime} \frac{\dot{\sigma}}{\dot{e}}\right) e+\eta \dot{e}^{v}\right\}$ 
and, by virtue of (2.8),

$\sigma=\frac{\rho}{\rho_{0}}\left\{\left(E+\eta \phi^{\prime \prime} \frac{\dot{\sigma}}{\dot{e}}\right) e+\eta \phi^{\prime}\right\}$

It is pointed out that, if $\eta=0$, this constitutive equation resembles closely its linearly elastic spring counterpart (2.4) which, however, assumes that the material density is not affected by the deformation.

The second term within the parenthesis appearing in right-hand side of either (3.2) or (3.3) reveals that this constitutive equation still preserves the non-linear differential equation features observed already in (2.11). However, further simplification becomes possible by next confining attention to the lowest polynomial form that the flow potential may attend without violation the initial condition (2.6b). This is the special case of a quadratic viscous flow potential,

$\phi(\sigma)=\frac{1}{2} G \sigma^{2}$,

where $G$ is some appropriate material modulus.

In that case, the viscous flow rule (2.8) yields

$\dot{e}^{v}=G \sigma$,

and enables (3.1) to obtain the special form

$W\left(e, \dot{e}^{v}\right)=\frac{1}{2} E e^{2}+\eta G e \sigma_{R}=\frac{1}{2} E e^{2}+\hat{\eta} e \sigma_{R} \geq 0$,

where use is made of the stress decomposition rule (2.10), along with the fact that, by definition, $\sigma_{D}$ contributes to dissipative work only.

It is also observed that the material parameters $\eta$ and $G$, introduced in (3.1) and (3.4), respectively, are here combined to form

$\hat{\eta}=\eta G$.

In what follows, this combined material parameter, $\hat{\eta}$, will thus be referred to as the generalised viscosity of the model associated with the quadratic flow potential (3.4). Introduction of the latter thus enables the constitutive equation (3.3) to obtain the form

$\sigma=\frac{\rho}{\rho_{0}}\left\{E e+\hat{\eta}\left(\sigma+\frac{\dot{\sigma}}{\dot{e}} e\right)\right\}$

which, due to involvement of the ratio $\dot{\sigma} / \dot{e}$, is still differential with respect to time as well as non-linear in both $\sigma$ and $e$.

\subsection{Energy and dissipation considerations}

By virtue of definition (2.9), the energy dissipation encountered during the described inelastic deformation is expected to be the following non-decreasing function of time:

$D^{w}=\int_{t_{0}}^{t} \psi \mathrm{d} t=\frac{1}{2} \int_{t_{0}}^{t} \sigma_{D} \dot{e}^{v} \mathrm{~d} t=\frac{G}{2} \int_{t_{0}}^{t} \sigma_{D} \sigma \mathrm{d} t \geq 0$, 
where $\sigma_{D}$ is to be determined, and the equality sign holds in the particular case that the spring behaves elastically $\left(\dot{e}^{v}=\sigma_{D}=0\right)$.

The following connection is then established between the internal energy (3.6) of the viscoelastic spring and the strain function (2.3) of its elastic counterpart:

$W^{e}(e)=W\left(e, \dot{e}^{v}\right)+D^{w}$.

By virtue of (3.9), this requires

$W^{e}(e) \geq W\left(e, \dot{e}^{v}\right) \geq 0$

which, through direct use of the internal energy expressions (2.3) and (3.6), as well the notion (3.7), leads to

$D^{w}=W^{e}(e)-W\left(e, \dot{e}^{v}\right)=-\hat{\eta} e \sigma_{R} \geq 0, \quad-\frac{1}{2} E e^{2} \leq \hat{\eta} e \sigma_{R} \leq 0$.

On the other hand, (3.10) also implies that the developed viscoelastic model may stop to represent a solid spring/fibre at some time instant, say $t=t_{F}>t_{0}$, that

$\left.D^{w}\right|_{t=t_{F}}=\left.\left.W^{e}\right|_{t=t_{F}} \Leftrightarrow W\left(e, \dot{e}^{v}\right)\right|_{t=t_{F}}=\left[\frac{1}{2} E e^{2}+\hat{\eta} e \sigma_{R}\right]_{t=t_{F}}=0$.

While the undeformed state, $e=0$, serves as an essentially trivial solution of this equation, the alternative solution

$\left[e / \sigma_{R}\right]_{t=t_{F}}=2|\hat{\eta}| / E$

estimates the ratio of the total strain and the work-recoverable part of stress at the time, $t=t_{F}$, that viscous effects may overwhelm the observed deformation and, hence, deprive from the spring its ability to store elastic energy.

\subsection{Determination of the elastic and inelastic parts of deformation and stress}

Solution of the simultaneous differential equations (2.5), (2.7) and (3.8), subject to the boundary and initial conditions (2.12) and (2.13), respectively, will enable determination of the displacement, the mass density and the stress distribution within the framework outlined so far in this section. With the stress, $\sigma$, and the total strain, $e$, being thus determined, and by virtue of (3.4), (2.14) will then provide the inelastic and elastic strain parts as well as the elastic part of the displacement as

$e^{v}=G \int_{t_{0}}^{t} \sigma \mathrm{d} t, \quad e^{e}=e-G \int_{t_{0}}^{t} \sigma \mathrm{d} t, \quad u^{e}=\int_{0}^{x} e^{e} \mathrm{~d} x=\int_{0}^{x}\left[e-G \int_{t_{0}}^{t} \sigma \mathrm{d} t\right] \mathrm{d} x$,

respectively. It is pointed out that expressions (3.15) do not guarantee that the total strain, $e$, and its resulting elastic and inelastic parts, $e^{e}$ and $e^{v}$, are necessarily all of the same sign.

The parts $\sigma_{D}$ and $\sigma_{R}$ of the stress that create dissipative and recoverable work, respectively, may then also be estimated in a couple of different ways. The direct and more accurate route followed later (see Sect. 5) is through time differentiation and subsequent comparison of (3.9) and (3.12a). This leads to the first of the relations

$\sigma_{D}=2 \frac{\dot{D}^{w}}{G \sigma}=-2 \eta \frac{\left(e \sigma_{R}\right)}{\sigma}, \quad \sigma_{R}=\sigma-\sigma_{D}$, 
the second being a result of the stress decomposition (2.10). It is anticipated that at least one of $\sigma_{D}$ and $\sigma_{R}$ should be of the same sign with $\sigma$. At this stage of the analysis though, no apparent reason seems to suggest that all three $\sigma, \sigma_{D}$ and $\sigma_{R}$ should be of the same sign throughout the spring span.

There is also provided in [21] an alternative approach for the estimation of $\sigma_{D}$ and $\sigma_{R}$. However, this will not be applied in the present problem of interest, as it is regarded as an approximate approach that may become applicable to more advanced/complicated generalised viscoelastic spring cases.

\section{Spatially homogeneous viscoelastic deformation}

It is now recalled that the deformation of a corresponding elastic spring is spatially homogeneous in the sense that, at any given time instance, $t$, the strain, $e$, is not only elastic, but also constant throughout the spring span (e.g. [20]). The same may not necessarily be true in the present viscoelastic spring case but, even if it is not, it can still be considered as an adequate approximation of the problem solution, at least for sufficiently small viscoelastic deformations.

Attention is accordingly now directed into the search for potential solutions of the outlined problem that are consistent with a displacement field of the form

$u=\left[\alpha(t)-\alpha_{0}\right] x, \quad \alpha_{0}=\alpha\left(t_{0}\right)$,

where $\alpha(t)$ is some suitable function of time that needs to be determined. This displacement pattern satisfies the boundary condition (2.12a) and underpins the homogeneous deformation field

$e=\left[\alpha(t)-\alpha_{0}\right], \quad \dot{e}=\dot{u}_{, x}=v_{, x}=\dot{\alpha}(t)$.

Moreover, it enables the continuity equation to obtain the form of the separable ordinary differential equation (ODE)

$\dot{\rho}+\rho \dot{\alpha}(t)=0$,

whose solution yields the material density of the spring as follows:

$\rho / \rho_{0}=\exp \left\{\alpha_{0}-\alpha(t)\right\}$

Introduction of these results into the constitutive Eq. (3.8) converts the latter into the following:

$\hat{\eta}\left(\alpha-\alpha_{0}\right) \dot{\sigma}+\left(\hat{\eta}-e^{\alpha-\alpha_{0}}\right) \dot{\alpha} \sigma=-\dot{\alpha} E\left(\alpha-\alpha_{0}\right)$.

Differentiation of this equation with respect to the spatial co-ordinate parameter, $x$, followed by appropriate use of the equation of motion (2.5), leads next to

$\hat{\eta}\left(\alpha-\alpha_{0}\right)\left(\dot{\rho} \ddot{u}_{, x}+\rho \dddot{u}_{, x}\right)+\left(\hat{\eta}-e^{\alpha-\alpha_{0}}\right) \dot{\alpha} \rho \ddot{u}_{, x}=0$,

and, by virtue of (4.1) and (4.4), to

$\hat{\eta}\left(\alpha-\alpha_{0}\right)(\dddot{\alpha}-\dot{\alpha} \ddot{\alpha})+\left(\hat{\eta} e^{\alpha_{0}-\alpha}-1\right) \dot{\alpha} \ddot{\alpha}=0$.

Potential solutions of this highly non-linear ODE will provide forms of the function $\alpha(t)$ that are consistent with the search for spatially homogeneous viscoelastic spring deformations. 
4.1 Steady (strictly quasi-static) viscoelastic deformation

Attention is next confined into the simplest time-dependent solution of (4.6), namely the linear function

$\alpha(t)=t / t_{0}$

This imposes conditions of steady viscoelastic deformation,

$e=t / t_{0}-1$

that takes place at constant speed, $v=1 / t_{0}$, and thus enables the equation of motion (2.5) to attain naturally its quasi-static form

$\sigma_{, x}=0$.

The stress thus is independent of position but, of course, is still dependent on time. By virtue of the boundary condition (2.12b), $\sigma$ is thus equal to the currently unknown boundary traction, $T(t)$, that drives the assumed deformation, (4.1) and (4.7). As it is considered unphysical for the magnitude of either $\sigma_{R}$ or $\sigma_{D}$ to exceed the magnitude of $T(t)$ at the boundary, it is accordingly concluded that both $\sigma_{R}$ and $\sigma_{D}$ have necessarily the same sign with $\sigma$ throughout the span of the spring.

Upon adopting at this point the reasonable assumption that the directions of total stress and total strain coincide throughout the spring span, at least within the small-strain regime, one concludes that this is also the case with the direction of either of $\sigma_{R}$ or $\sigma_{D}$. It is thus seen that

$\sigma_{R} e \geq 0$

and as (3.12) thus requires

$\hat{\eta}=\eta G<0$

the material moduli $\eta$ and $G$ should be having opposite signs.

It follows by virtue of (3.5) that, if

$G>0, \quad \eta<0$,

then a spring element in extension $(\sigma>0)$ experiences inelastic strain of an increasing rate, while in compression $(\sigma$ $<0)$ the rate of inelastic strain will be decreasing. This effect is evidently reversed it in the alternative case, where

$G<0, \quad \eta>0$.

However, as $\sigma$ equals the boundary traction throughout the spring span, the whole spring is either in extension or in compression, and this thus clearly depends on the direction of $T(t)$. For simplicity, and by virtue of the $T$-direction shown in Fig. 1, only the tensile spring problem will be considered in what follows, although most, if not all of the outlined analytical results hold regardless of whether the spring is in tension or in compression. Moreover, wherever detailed specification of $G$ and $\eta$ is required for numerical applications, only the case (4.12) will be considered. This is because, as is shown later in Sect. 5 with the help of an Appendix, consideration of the alternative case (4.13) leads to values of $\sigma_{R}$ that violate the necessary condition (4.10). 
Fig. 2 Non-dimensional total stress parameter $\bar{\sigma}=\sigma / E$ against total strain $e$ for several values of the generalised viscosity parameter $|\hat{\eta}|=|G \eta|(|\hat{\eta}|=0$ corresponds to an elastic spring)

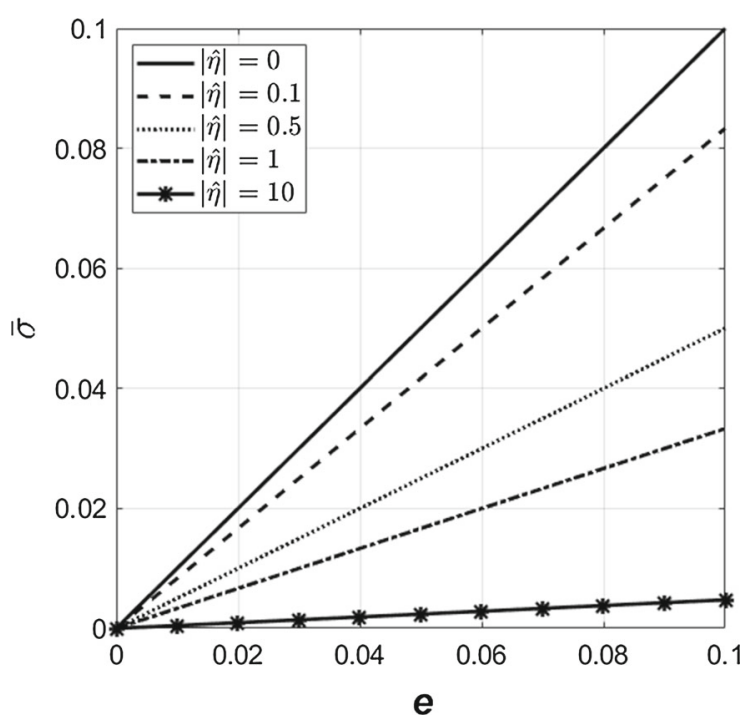

Under these considerations, the constitutive equation (3.8) acquires the form of the linear first-order ODE,

$\dot{\sigma}+p(t) \sigma=q(t)$

where

$p(t)=\frac{\hat{\eta}-\mathrm{e}^{t / t_{0}-1}}{\hat{\eta} t_{0}\left(t / t_{0}-1\right)}, \quad q(t)=-\frac{E}{\hat{\eta} t_{0}}$.

Solution of this equation, subject to the initial condition (2.13b), provides the stress in the closed form

$\sigma=\frac{1}{I(t)} \int_{t_{0}}^{t} I(t) q(t) \mathrm{d} t, \quad I(t)=\exp \left(\int p(t) \mathrm{d} t\right)$,

where, however, the noted integrations may be accomplished only numerically.

Nevertheless, an alternative form of this solution is possible, and this is provided in Appendix B, in the form (B.1) of an infinite power series expansion in $e$. The coefficients of that series are given by the recurrence formula (B.5), which reveals features of very fast convergence within the regime of small strain. As a matter of fact, the first term of that series is proportional to $e$ and, as the recurrence formula shows that the second term is of the order of $e^{2}$, the order of each subsequent term decreases very rapidly.

A single term truncation of (B.1), namely

$\sigma \simeq \hat{E} e, \quad \hat{E} \equiv \sigma_{1}=\frac{E}{1+2|\hat{\eta}|}$,

then provides an excellent approximation of the exact solution (4.16), in the sense that it produces numerical results that are practically unaffected by the contribution of the truncated higher-order terms. Indeed, the straight lines depicted in Fig. 2 are all based on (4.17) and remain unaffected if re-plotted by retaining more terms in (B.1). As will be seen later in Sect. 5 though, the corresponding work-recoverable and energy-dissipative stress parts vary with the strain in a non-linear manner. 
Fig. 3 Inelastic strain $e^{v}=\lambda e^{2}$ against total strain $e$ for several positive values of the normalisation value $\lambda=e^{-1} \neq \exp (-1)$ is used only for graphical incorporation of the straight bisector of the co-ordinate axes) parameter $\lambda$ (the variable

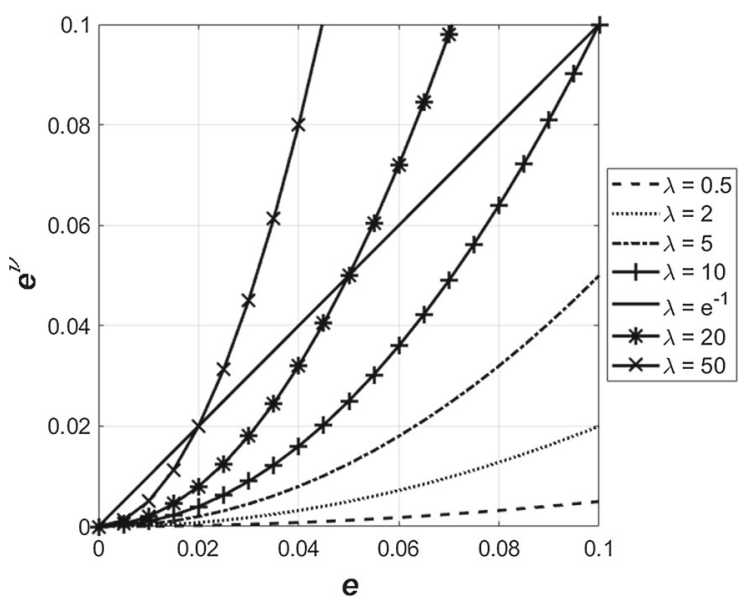

Fig. 4 Elastic strain

$e^{e}=e-e^{v}=(1-\lambda e) e$ against total strain $e$, for the four lower values of $\lambda$ employed in Fig. 3 (the variable value $\lambda=e^{-1}$ $\neq \exp (-1)$, is used only for graphical incorporation of the straight bisector of the co-ordinate axes)

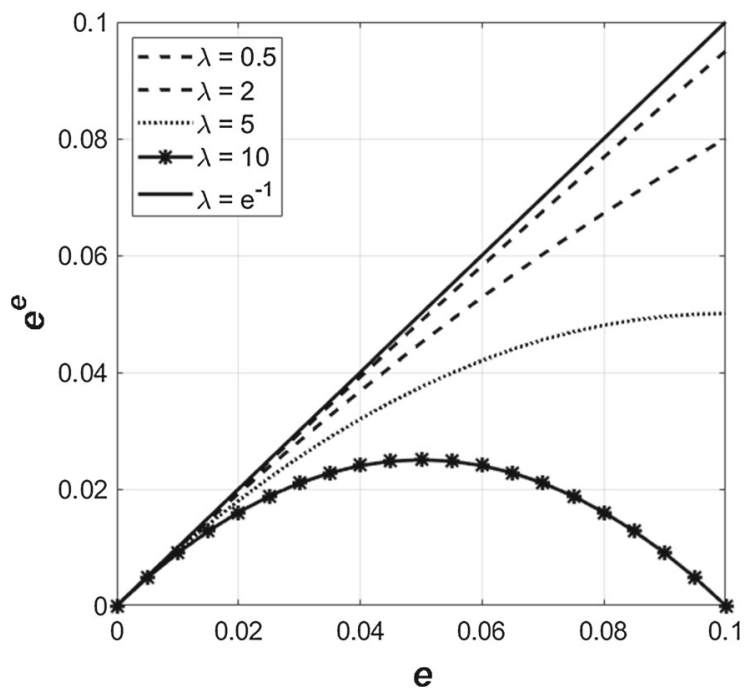

\subsection{Relevant numerical results and discussion}

For all numerical results shown in what follows, the varying values of $e$ are considered positive to comply with the nature of the tensile spring deformation implied earlier in Fig. 1. Moreover, care is taken for the total strain, $e$, to vary within reasonably small bounds. It is pointed out in this regard that the so-called infinitesimal strain regime may be narrower to the small-strain range employed in this communication, as is sometimes subjected to an upper-bound restriction that does not exceed 5\%. Nevertheless, the infinitesimal strain regime is still part of the small-strain range employed in what follows and, if necessary, the associated discussion and relevant observations can be restricted accordingly. It should be emphasised though that a decrease or even an increase of the small strain upper limit implied in Figs. 2, 3, 4 and 5 leaves completely unaffected the qualitative features of the presented numerical results.

Under these considerations, the straight lines depicted in Fig. 2 exhibit the practically linear manner that the non-dimensional stress parameter

$\bar{\sigma}=\sigma / E=e /(1+2|\hat{\eta}|)$ 
Fig. 5 Elastic strain

$e^{e}=1-e^{v}=(1-\lambda e) e$

against total strain $e$, for the

three higher values of $\lambda$

employed in Fig. 3

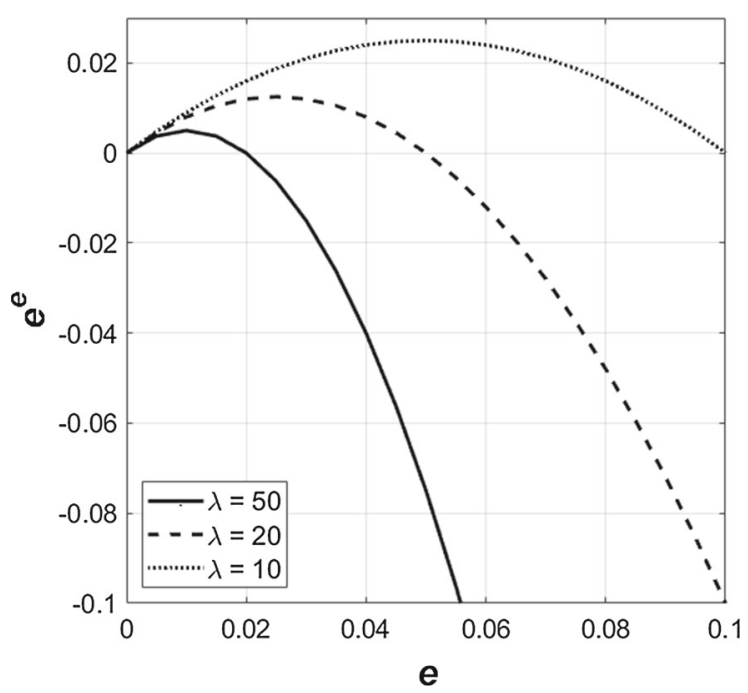

varies with increasing the total strain $e$, for several values of the viscosity parameter $|\hat{\eta}|$. Figure 2 thus clearly demonstrates the decreasing effect that increasing viscosity exerts on the stress value.

It is emphasised that the non-dimensionalisation employed in (4.18) is based on the Young modulus, $E$, of the corresponding elastic spring/fibre, rather than its effective viscoelastic spring counterpart, $\hat{E}$, defined in (4.17b). As a matter of fact, if $E$ were replaced in (4.18) by $\hat{E}$, then all straight lines shown in Fig. 2 would appropriately rotate and become identical with their depicted elastic spring counterpart, obtained by setting in (4.18) $|\hat{\eta}|=0$. It becomes thus understood that any set of numerical results involving below the non-dimensional stress parameter $\sigma / \hat{E}$ is similarly a contracted representation of a wider set of relevant results.

In view of the stress distributions depicted in Fig. 2, it may erroneously be felt at this point that the effect of viscosity is confined within a simple replacement of the actual spring modulus, $E$, by its effective counterpart, $\hat{E}$, or, equivalently, the replacement of a linearly elastic spring/fibre with another that possesses some appropriately reduced elastic modulus. However, as will be seen in what follows, the present viscoelastic model provides also a considerable amount of further interesting information regarding the manner that (i) the total strain splits into elastic and inelastic parts, (ii) the total stress splits into work-recoverable and energy-dissipative parts, and (iii) the spring internal energy is accumulated or dissipated during the assumed viscoelastic deformation.

Under these considerations, the inelastic and the elastic parts of the total strain $e$, as well as the elastic part of the total displacement, are next obtained by inserting (4.18) into (3.15) and performing the noted integrations. These are as follows:

$e^{v}=\lambda e^{2}, \quad e^{e}=(1-\lambda e) e, u^{e}=(1-\lambda e) e x$,

where

$\lambda=\frac{E G t_{0}}{2(1+2|\hat{\eta}|)}=\frac{\hat{E} G t_{0}}{2}$,

appears naturally at this stage of the solution as a dimensionless, inelastic strain normalisation parameter. This parameter has necessarily the same sign with $G$ and may thus be either positive or negative. However, it has already 
been anticipated that only choices of $G$ and $\eta$ that comply with (4.12) enable satisfaction of the necessary condition (4.10) and, hence, someone can safely consider that

$\lambda>0, \quad \eta<0$,

in what follows.

For several values of the normalisation parameter $\lambda$, Fig. 3 thus demonstrates the parabolic manner that the inelastic part of the strain, $e^{v}$, varies with increasing the total strain, $e$; see also (4.19a) and (4.8), respectively. It is observed that, for relatively small values of $\lambda$, the inelastic part of strain remains smaller than $e$. This is the case for the three lower $\lambda$-values, for each one of which Fig. 4 depicts further the corresponding variation of the elastic strain part; the $e^{e}$ variation that corresponds to each of the three higher values of $\lambda$ is similarly depicted in Fig. 5 . For the low $\lambda$-values, Figs. 3 and 4 thus show that both $e^{v}$ and $e^{e}$ remain positive and, therefore, tensile within the employed region of total strain variation.

However, for the three higher values of $\lambda$, there comes a time instance that the inelastic strain reaches the value of $e$ and, hence, the elastic strain becomes zero. This evidently happens when the relevant parabolic curve reaches the bisector of the co-ordinate axes depicted in Fig. 3. Beyond that crossing point, the positive value of the inelastic strain tends to exceed its total strain counterpart $\left(e^{v}>e\right)$, as is demonstrated in Fig. 3 for the two higher values of $\lambda$. However, despite the temporary zero value that $e^{e}$ attains at that point, the elastic part of the deformation is still active due to the non-zero value of the strain energy stored already in the material. Figure 5 thus shows that when the spring is loaded beyond the implied crossing point, the stored strain energy enables the elastic strain to become compressive (change of sign) and to thus counteract the tensile part of $e^{v}$ that tends to exceed the total $e$-value.

\section{Strain energy and energy dissipation: work-recoverable and energy-dissipative parts of stress}

The form (3.6) of the strain energy function implies that determination of the energy stored in the deforming viscoelastic spring requires previous evaluation of the stress part $\sigma_{R}$ that produces recoverable/elastic work. It is accordingly observed that a combination of (3.5) with (3.9) leads to

$\dot{D}^{w}=\psi=\frac{1}{2} \sigma_{D} \dot{e}^{v}=\frac{G}{2}\left(\sigma-\sigma_{R}\right) \sigma$,

where use is also made of (2.10).

On the other hand, time differentiation of (3.12a) yields

$\dot{D}^{w}=-\hat{\eta}\left(e \sigma_{R}\right)$

Equating the right-hand sides of (5.1) and (5.2), and making also use of (4.11), one obtains

$\eta e \dot{\sigma}_{R}+\left(\eta \dot{e}-\frac{1}{2} \sigma\right) \sigma_{R}=-\frac{1}{2} \sigma^{2}$

With use of (4.8) and (4.17a), this equation acquires the alternative form

$\dot{\sigma}_{R}+\left(\frac{1}{t-t_{0}}-\frac{\hat{E}}{2 \eta}\right) \sigma_{R}=-\frac{\hat{E}^{2}}{2 \eta}\left(t / t_{0}-1\right)$.

This is essentially in the standard form (4.14) of a first-order linear ODE and, hence, (5.4) it also susceptible to the standard closed form solution (4.16). 
Nevertheless, an equivalent power series solution of (5.4) is obtained in Appendix C. This is practically again more useful than (4.16), because (i) it makes it clear that only the case (4.12) needs to be considered in this investigation, (ii) enables formation of the following closed form relationship between the total stress and its work-recoverable counterpart:

$\sigma_{R} / \sigma=-\frac{2}{(\hat{\lambda} e)^{2}}\left(\sum_{n=0}^{\infty} \frac{(-\hat{\lambda} e)^{n}}{n !}-1+\hat{\lambda} e-\frac{(\hat{\lambda} e)^{2}}{2}\right)=2\left(\frac{1-\exp (-\hat{\lambda} e)}{(\hat{\lambda} e)^{2}}-\frac{1}{\hat{\lambda} e}+\frac{1}{2}\right)$,

and (iii) thus makes evident the natural appearance of the new, non-dimensional normalisation parameter

$\hat{\lambda}=\frac{\hat{E} t_{0}}{2|\eta|}=\frac{E G t_{0}}{2|\hat{\eta}|(1+2|\hat{\eta}|)}=\lambda /|\hat{\eta}|>0$.

It is pointed out that, by virtue of (3.11) and (4.12), the larger (smaller) is the positive value of $\hat{\lambda}$, the stronger is the influence of the elastic (inelastic) effects of the deformation on the work-recoverable part of the stress.

The fact that both $\sigma_{R}$ and $\sigma_{D}$ have already been anticipated tensile and, therefore, positive enables (2.10) to provide the condition:

$\sigma_{R} / \sigma<1$.

If connected with (5.5), this inequality provides from the range of values of $\hat{\lambda} e$ to satisfy the following condition:

$\frac{1}{\hat{\lambda} e}\left[\frac{1-\exp (-\hat{\lambda} e)}{\hat{\lambda} e}-1\right]<0$.

It can readily be verified that this condition is indeed satisfied in the present case of interest, where both $\hat{\lambda}$ and $e$ are considered positive.

By virtue of (2.10) and (5.5), the energy-dissipative part of the stress relates with the total stress as follows:

$\sigma_{D} / \sigma=\frac{2}{\hat{\lambda} e}\left(1-\frac{1-\exp (-\hat{\lambda} e)}{\hat{\lambda} e}\right)>0$.

Use of (5.5) and (5.9) thus enables Fig. 6 to demonstrate the highly non-linear manner that the ratios $\sigma_{R} / \sigma$ and $\sigma_{D} / \sigma$ vary within a substantially large region of $\hat{\lambda} e$-values.

The curves drawn in that figure make it evident that inelastic effects dominate in regions of relatively small $\hat{\lambda} e-$ values. However, their influence is continually degreasing with increasing $\hat{\lambda} e$ and is dying out with $\hat{\lambda} e$ approaching infinity. Elastic effects thus take gradually over and become dominant for larger values of the $\hat{\lambda} e$-parameter.

It is next seen that, with use of (5.5), (4.7) and (4.11), the internal energy function (3.6) may take the form

$W\left(e, \dot{e}^{v}\right)=\frac{1}{2} E^{v} e^{2}, \quad E^{v}=E\left\{1+\frac{4|\hat{\eta}|}{1+2|\hat{\eta}|}\left(\frac{1-\hat{\lambda} e-\exp (-\hat{\lambda} e)}{(\hat{\lambda} e)^{2}}+\frac{1}{2}\right)\right\}$,

which, by virtue of (5.8), is clearly a decreasing function of the strain, $e$. This expression demonstrates the manner that the viscoelastic spring modulus, $E^{v}$, changes with the deformation. 
Fig. 6 Variation of the ratios $\sigma_{R} / \sigma$ and $\sigma_{D} / \sigma$ throughout a substantially large region of the $\hat{\lambda} e$-parameter $(0 \leq \hat{\lambda} e<\infty)$

Fig. 7 Variation of the ratio $W\left(e, \dot{e}^{v}\right) / W^{e}$ with increasing the $\hat{\lambda} e$-parameter $(0<\hat{\lambda} e<\infty)$, for several values of the generalised viscosity parameter, $\hat{\eta}<0$
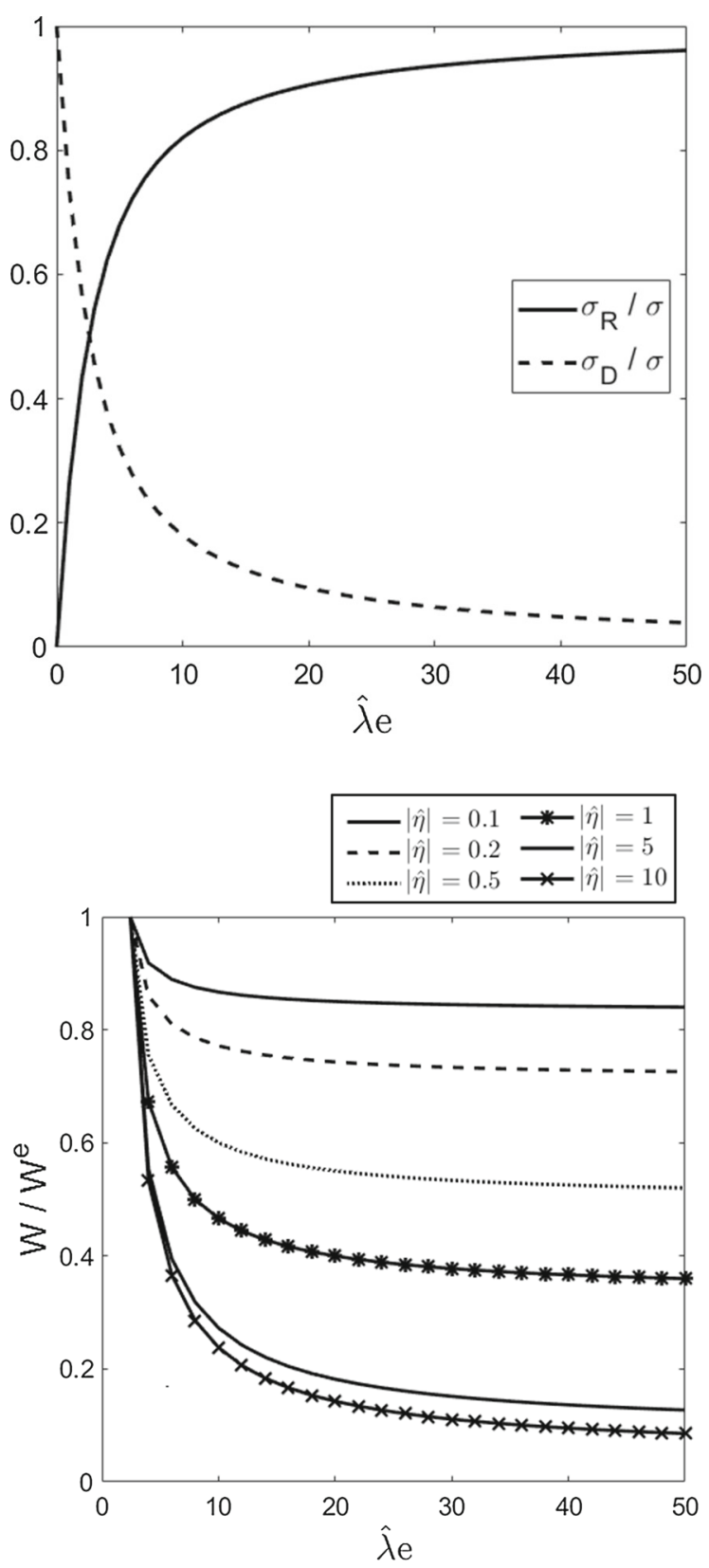

Moreover, (5.10) may alternatively be written in terms of the strain energy function (2.3) of the corresponding elastic string/fibre as follows:

$W\left(e, \dot{e}^{v}\right)=\left\{1+\frac{4|\hat{\eta}|}{1+2|\hat{\eta}|}\left(\frac{1-\hat{\lambda} e-\exp (-\hat{\lambda} e)}{(\hat{\lambda} e)^{2}}+\frac{1}{2}\right)\right\} W^{e}$.

Based on this formula, Fig. 7 demonstrates that manner that the ratio $W\left(e, \dot{e}^{v}\right) / W^{e}$ decreases with increasing the $\hat{\lambda} e$-parameter, for several values of the generalised viscosity parameter, $\hat{\eta}$. 
Fig. 8 Variation of the ratio $W\left(e, \dot{e}^{v}\right) / W^{e}$ in the vicinity of $\hat{\lambda} e=0$ for the values of $\hat{\eta}$ employed in Fig. 7

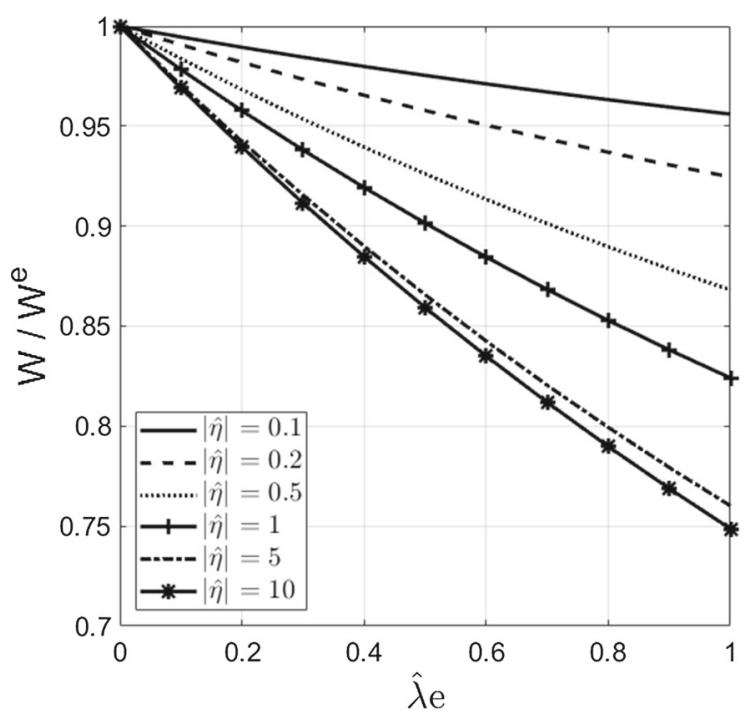

It is pointed out that, at the limiting case that $\hat{\lambda} e$ approaches zero, a double application of L' Hospital's rule reveals that

$\lim _{\hat{\lambda} e \rightarrow 0} \frac{1-\hat{\lambda} e-\exp (-\hat{\lambda} e)}{(\hat{\lambda} e)^{2}}=-1$,

and, hence, that the value of the ratio $W\left(e, \dot{e}^{v}\right) / W^{e}$ is everywhere finite and well defined. However, a direct computational evaluation of the large ratio-expression appearing in (5.11) creates the wrong impressions that the left-hand side of (5.12) is indeterminate. The resulting numerical instability thus prevents the graphical user interface employed for creation of the plots depicted in Fig. 7 from producing reliable numerical results in the vicinity of $\hat{\lambda} e$ $=0$.

However, the numerical results depicted in Fig. 8 make it clear that nothing unusual, unexpected or, more generally, singular takes place at the vicinity of $\hat{\lambda} e=0$. These numerical results (Fig. 8) hold within in the chosen, relatively small-value region of $\hat{\lambda} e(0 \leq \hat{\lambda} e \leq 1)$ for identical material parameters to those employed in Fig. 7 . However, the depicted curves of the ratio $W\left(e, \dot{e}^{v}\right) / W^{e}$ are drawn through use of a four-term truncation of the series solution (C.6) of (5.4), rather than its mathematically equivalent closed form (5.5).

Under these considerations, it is safe for someone to consider that all curves depicted in Fig. 7 depart from the top left corner of that figure. Hence, either of (5.10) or (5.11) can finally reveal that the value of the corresponding dissipated energy is

$D^{w}=-\frac{4|\hat{\eta}|}{1+2|\hat{\eta}|}\left(\frac{1-\hat{\lambda} e-\exp (-\hat{\lambda} e)}{(\hat{\lambda} e)^{2}}+\frac{1}{2}\right) W^{e}>0$,

as can alternatively be verified by combining (5.5) and (3.12a). 


\section{Further discussion and conclusions}

It is emphasised that the steady viscoelastic spring/fibre deformation considered in Sect. 4, and discussed afterwards extensively is Sect. 5, becomes possible only when the boundary condition (2.12b) is applied in consistence with (4.17) and, therefore, when the externally applied tensile traction evolves in time as follows:

$T(t) \approx \frac{E}{1+2|\hat{\eta}|} e=\frac{E}{1+2|\hat{\eta}|}\left(t / t_{0}-1\right)$.

The relevant solution detailed in Sect. 4 then represents an exceptional viscoelastic spring/fibre type of deformation that is followed neither by stress relaxation nor by strain creep. It is, however, recalled that the stress relaxation or strain creep behaviour of a standard spring-dashpot viscoelastic constitutive model is usually studied by subjecting the model into some known, usually constant amount of strain or stress, respectively (e.g. [23-25]).

If such an essentially arbitrary loading condition is similarly applied externally on the present viscoelastic model, the constitutive law (3.8) will evidently fail to respond in a manner consistent with the equation of motion (2.5) or the continuity equation (2.7). It is in fact anticipated that, even if the spatially homogenous deformation conditions (4.1) were potentially applicable in such a case, the constitutive law (3.8) would probably fail to satisfy the alternative relevant form (4.6) attained in that case by the equation of motion. Most of these comments and observations hold evidently true in relevant mathematical models that do not introduce quasi-static conditions leading to (4.9) in the strict manner considered in this communication, but still employ it, in the usual manner, as a reliable approximation of corresponding boundary value problems (see also [26]).

The thus emerging equilibrium unbalance would then set up a stress relaxation and/or a strain creep process that would continue until mass density balance and stress equilibrium were both completely restored. It is accordingly concluded that the exact viscoelasticity solution presented in Sect. 4 is further expected to represent the final stage of stress relaxation and/or strain creep that follow any relevant small-strain viscoelastic deformations that takes earlier place in some unsteady (not quasi-static) manner.

Alternatively, an initially unsteady small-strain viscoelastic deformation that conforms with the spatially homogeneous deformation assumptions considered at the beginning of Sect. 4 should be followed by some appropriate stress relaxation and/or strain creep process that would enable it to approach as close as possible either (i) the relevant steady equilibrium state detailed in Sect. 4.1 or (ii) some alternative, nearer to it equilibrium state that, if available, will also satisfy the continuity equation (2.7) and the reduced form (4.6) of the equation of motion.

It is further observed with interest that, despite its non-linear form, the constitutive equation (3.8) can be rearranged in the following alternative form:

$\sigma=\tilde{E} e, \tilde{E}=\frac{\left(E+\hat{\eta} \frac{\dot{\sigma}}{\dot{e}}\right)}{\left(\frac{\rho_{0}}{\rho}-\hat{\eta}\right)}$

This resembles closely either its well-known linear elastic spring/rod counterpart (2.4) where, however, the appearing elastic modulus, $E$, is constant, or its homogeneous, steady deformation viscoelastic approximation (4.17), where the effective (or reduced) constant modulus $\hat{E}$ emerges as an excellent approximation of the essentially unknown time-dependent modulus $\tilde{E}$ implied in (6.2).

In the present case though, the value of the effective Young's modulus $\hat{E}$ depends on the generalised viscosity parameter $\hat{\eta}$. In this regard, it is remarkable that the deformation-dependent modulus $\tilde{E}$ appearing in (6.2) is so accurately approximated in (4.17) by the its constant counterpart $\hat{E}$. It is in fact remarkable that, in the small-strain regime of interest, (4.17) replaces so accurately the essentially non-linear deformation response of a viscoelastic spring with what, at a first view, might be thought of as the response of a linearly elastic spring (see also the relevant straight lines depicted in Fig. 2 for $|\hat{\eta}| \neq 0$ ). 
Nevertheless, the more thorough relevant investigation performed in Sects. 4 and 5 revealed that this seemingly linear material behaviour is composed by well-specified elastic and inelastic parts whose relevant strain and stress states vary non-linearly with the implied small-strain viscoelastic deformation. Moreover, the analysis detailed in Sect. 5 made possible a mathematically exact determination of the dissipated energy and the work stored in the material during the implied deformation process.

It may still be pointed out though that the analytical solution detailed in Sect. 4, as well as all relevant numerical results presented and discussed afterwards, refer only to continuous loading conditions, starting from an initial state at which the spring/fibre is both unstressed and unstrained. The fact that no relevant unloading process was considered is due to that such a process may be defined, in advance, in one of several different ways. Indeed, even in the simple case that such a spring unloading process is anticipated elastic, someone still needs to specify the relevant constant value of the spring/fibre elastic modulus.

Choosing, for instance, any of the straight lines depicted in Fig. 2 for $|\hat{\eta}| \neq 0$ and assuming that unloading from some point of that line takes place elastically, one has still to assign a value to the corresponding elastic (Young's) modulus. If this is assumed equal to the value $\hat{E}$ reached already during the outlined viscoelastic loading process, then elastic unloading will evidently take place by tracing the same line "downwards", in the opposite direction. If, on the other hand, there is some reason (such as experimental evidence) suggesting that elastic unloading takes place under the influence of the modulus $E$ of the corresponding "virgin" elastic spring, then unloading would trace downwards a straight line parallel to that depicted in Fig. 2 for $|\hat{\eta}|=0$.

The choice of any other elastic or viscoelastic, spatially homogeneous, steady unloading process would end up with use of some different effective or reduced Young's modulus and would thus trace downwards some different straight line. In case that such an unloading process is considered viscoelastic, the slope of that line would evidently depend not only on the anticipated value of the Young's modulus but also on the value of its associated generalised viscosity parameter, $\hat{\eta}$. It is anticipated that, while the fibre will still be in tension during unloading, either of these material moduli may not necessarily be identical to their counterparts employed during the corresponding loading process.

Under these considerations, it is mentioned in passing that by (i) keeping $E$ constant during several successive loading-unloading cycles of a relevant deformable spring/fibre and, as an effect of each of those cycles, (ii) decreasing successively the influence of the generalised viscosity parameter $\hat{\eta}$, a constitutive equation of the type (3.8) may succeed to model successfully the Mullins hardening effect observed in the behaviour of several kinds of polymers and standard rubber-like materials (e.g. [27-29]). However, at these early modelling stages, this observation is only regarded as a possibility that requires thorough investigation and further study. This is at least because such a further study/investigation will certainly require use of a relevant constitutive equation that is valid within the large rather than the small strain/deformation regime (see also [21]).

It is noted in this context that the linearly inelastic spring model described in Sect. 4, and the corresponding analytical solution detailed in Sect. 5, may well guide the search for relevant large deformation solutions associated with, and stemming from a proper and detailed consideration of the fully non-linear inelastic spring model detailed in [21]. Such solutions are of paramount importance for a better understanding of the large inelastic deformation of one-dimensional structures, though may still be considered inadequate for relevant understanding of more realistic, three-dimensional inelastic material behaviour. Nevertheless, three-dimensional models that correspond to the nonlinear inelastic spring modelled in [21] are already available in the literature [30-32] and are expected to receive considerable benefit and boost from any relevant one-dimensional progress, such as the present one, that becomes gradually available.

This is because the non-linear spring model developed in [21] and, hence, its small stain version considered in the present communication, may alternatively be obtained by specialising in one-dimension its three-dimensional counterpart detailed in [32]. It is recalled in this regard [21] that, by considering that the new plasticity-type postulations detailed in [32] are relevant and applicable to classical viscoelasticity situations, someone can clearly observe that all three subjects of Hyperelasticity, Plasticity and Viscoelasticity share a common theoretical background. The here presented small-strain one-dimensional model, analysis and relevant solution may thus be considered as the simplest possible example that fits within this larger, and still potential theoretical unification picture. 
Acknowledgements The author gratefully acknowledges the technical assistance received from Dr Mustafa Arda, Department of Mechanical Engineering, Trakya University, Turkey.

Open Access This article is licensed under a Creative Commons Attribution 4.0 International License, which permits use, sharing, adaptation, distribution and reproduction in any medium or format, as long as you give appropriate credit to the original author(s) and the source, provide a link to the Creative Commons licence, and indicate if changes were made. The images or other third party material in this article are included in the article's Creative Commons licence, unless indicated otherwise in a credit line to the material. If material is not included in the article's Creative Commons licence and your intended use is not permitted by statutory regulation or exceeds the permitted use, you will need to obtain permission directly from the copyright holder. To view a copy of this licence, visit http://creativecommons.org/licenses/by/4.0/.

Open Access This article is licensed under a Creative Commons Attribution 4.0 International License, which permits use, sharing, adaptation, distribution and reproduction in any medium or format, as long as you give appropriate credit to the original author(s) and the source, provide a link to the Creative Commons licence, and indicate if changes were made. The images or other third party material in this article are included in the article's Creative Commons licence, unless indicated otherwise in a credit line to the material. If material is not included in the article's Creative Commons licence and your intended use is not permitted by statutory regulation or exceeds the permitted use, you will need to obtain permission directly from the copyright holder. To view a copy of this licence, visit http://creativecommons.org/licenses/by/4.0/.

\section{Appendix A: Direct derivation of the constitutive equation (2.11)}

The rate of the internal energy that the elastic part of the deformation accumulates in an arbitrary part $[0, x]$ of the deforming spring/fibre is governed by the usual rate-of-energy equation

$$
\frac{\mathrm{d}}{\mathrm{d} t} \int_{0}^{x} \rho\left(\frac{1}{\rho_{0}} W+\frac{1}{2} \dot{v}^{2}\right) \mathrm{d} x=\sigma v
$$

where $W$ is the internal energy (2.6) per unit length. It is recalled that, unlike the relevant linear elasticity postulate that endorses negligible change of material density, the latter is here regarded as potentially influential due to the inelastic part of the deformation. Hence, use of the rule [22]

$$
\frac{\mathrm{d}}{\mathrm{d} t} \int_{0}^{x} \rho\left(\frac{1}{\rho_{0}} W+\frac{1}{2} \dot{v}^{2}\right) \mathrm{d} x=\int_{0}^{x} \rho \frac{\mathrm{d}}{\mathrm{d} t}\left(\frac{1}{\rho_{0}} W+\frac{1}{2} \dot{v}^{2}\right) \mathrm{d} x,
$$

followed by differentiation with respect to $x$, leads to

$$
\frac{\rho}{\rho_{0}} \dot{W}=\left(\sigma_{, x}-\rho \dot{v}\right) v+\sigma v_{, x} .
$$

On the other hand, differentiation of (2.6a) with respect to time leads to

$$
\dot{W}=\frac{d W}{d t}=\frac{\partial W}{\partial e} \dot{e}+\frac{\partial W}{\partial \dot{e}^{v}} \ddot{e}^{v}=\left(\frac{\partial W}{\partial e}+\frac{\partial W}{\partial \dot{e}^{v}} \frac{\ddot{e}^{v}}{\dot{e}}\right) \dot{e}=\left(\frac{\partial W}{\partial e}+\frac{\partial W}{\partial \dot{e}^{v}} \frac{\ddot{e}^{v}}{\dot{e}}\right) v_{, x} .
$$

A comparison of this result with (A.3) yields

$$
\left(\sigma_{, x}-\rho \dot{v}\right) v+\left[\sigma-\frac{\rho}{\rho_{0}}\left(\frac{\partial W}{\partial e}+\frac{\partial W}{\partial \dot{e}^{v}} \frac{\ddot{e}^{v}}{\dot{e}}\right)\right] v_{, x}=0
$$

Due to the arbitrariness of both $v$ and $v_{, x}$, this leads to the equation of motion (2.5) and to the constitutive equation (2.11), where use is also made of (2.8). 


\section{Appendix B: Power series solution of the ODE (4.14)}

A power series solution of (4.14) is sought, in the form

$\sigma(t)=\sum_{n=1}^{\infty} \sigma_{n}\left(t / t_{0}-1\right)^{n}=\sum_{n=1}^{\infty} \sigma_{n} e^{n}$

where the constant coefficient $\sigma_{n}$ are to be determined. Such a potential solution evidently satisfies the initial condition (2.13b).

Upon inserting (B.1) into (4.14), one initially obtains

$\sum_{n=1}^{\infty} \frac{n}{t_{0}} \sigma_{n}\left(t / t_{0}-1\right)^{n-1}+\left\{\frac{1}{t_{0}\left(t / t_{0}-1\right)}-\frac{e^{t / t_{0}-1}}{t_{0} \hat{\eta}\left(t / t_{0}-1\right)}\right\} \sum_{n=1}^{\infty} \sigma_{n}\left(t / t_{0}-1\right)^{n}=-\frac{E}{\hat{\eta} t_{0}}$.

This is further seen equivalent to the following equation:

$\sum_{n=1}^{\infty}(n+1) \sigma_{n}\left(t / t_{0}-1\right)^{n-1}+\frac{e^{t / t_{0}-1}}{|\hat{\eta}|\left(t / t_{0}-1\right)} \sum_{n=1}^{\infty} \sigma_{n}\left(t / t_{0}-1\right)^{n}=\frac{E}{|\hat{\eta}|}$,

where the fact that $\hat{\eta}<0$ is also taken into consideration; see (4.11).

After replacing the appearing exponential term with its standard Taylor-series expansion,

$e^{t / t_{0}-1}=\sum_{m=0}^{\infty} \frac{\left(t / t_{0}-1\right)^{n}}{n !}$

(B.3) is finally found equivalent with the following equation:

$\sum_{\ell=0}^{\infty}\left\{(\ell+2)+\frac{1}{|\hat{\eta}|} \sum_{m=0}^{\infty} \frac{1}{m !}\left(t / t_{0}-1\right)^{m}\right\} \sigma_{\ell+1}\left(t / t_{0}-1\right)^{\ell}-\frac{E}{|\hat{\eta}|}=0$.

Hence, by collecting like powers of $\left(t / t_{0}-1\right)$ and requiring from their coefficients to be equal to zero, one finds that

$\sigma_{1}=\frac{E}{1+2|\hat{\eta}|}, \quad \sigma_{k}=\frac{-1}{1+(k+1)|\hat{\eta}|} \sum_{j=1}^{k-1} \frac{\sigma_{j}}{(k-j) !} \quad(k \geq 2)$.

\section{Appendix C: Power series solution of the ODE (5.4)}

The power series solution sought for (5.4) is of the form

$\sigma_{R}(t)=\sum_{n=1}^{\infty} \hat{\sigma}_{n}\left(t / t_{0}-1\right)^{n}=\sum_{n=1}^{\infty} \hat{\sigma}_{n} e^{n}$

where the appearing constant coefficients $\hat{\sigma}_{n}$ are to be determined. Such a series expansion of $\sigma_{R}$ is consistent with the initial condition (2.13b) in the sense that, by virtue of (2.10), still enables the total stress (B.1) to satisfy that initial condition. 
Hence, introduction of (C.1) into (5.4) yields

$2 \hat{\sigma}_{1}+\sum_{n=1}^{\infty}\left[(n+2) \hat{\sigma}_{n+1}-\frac{\hat{E} t_{0}}{2 \eta} \hat{\sigma}_{n}\right]\left(t / t_{0}-1\right)^{n}=-\frac{\hat{E}^{2} t_{0}}{2 \eta}\left(t / t_{0}-1\right)$.

By initially collecting and, afterwards, equating to zero coefficients of like powers of $\left(t / t_{0}-1\right)$, one thus obtains

$\hat{\sigma}_{1}=0, \quad \hat{\sigma}_{2}=-\frac{\hat{E}^{2} t_{0}}{6 \eta}, \quad \hat{\sigma}_{k+1}=\frac{\hat{E} t_{0}}{2(k+2) \eta} \hat{\sigma}_{k} \quad(k \geq 2)$.

These formulas make it clear that, if $\eta>0$, then $\hat{\sigma}_{k}<0$ for all $k \geq 2$ and, hence, (C.1) predicts that $\sigma_{R}<0$. However, as such a result would violate the necessary condition (4.10), $\eta$ is necessarily negative and, therefore, only that case (4.12) needs to be considered in this investigation. In that case though, (C.3) may obtain the following alternative form

$\hat{\sigma}_{1}=0, \hat{\sigma}_{2}=\frac{\hat{E}^{2} t_{0}}{6|\eta|}, \quad \hat{\sigma}_{k+1}=-\frac{\hat{E} t_{0}}{2(k+2)|\eta|} \hat{\sigma}_{k} \quad(k \geq 2)$,

thus showing that (C.1) is an alternating series.

It can accordingly be readily verified that, when (4.12) holds, the power series solution sought for the (5.4) takes the alternating series form

$\sigma_{R}=-2 \hat{E} \sum_{n=2}^{\infty} \frac{(-1)^{n-1} \hat{\lambda}^{n-1}}{(n+1) !}\left(t / t_{0}-1\right)^{n}=-2 \hat{E} \sum_{n=2}^{\infty} \frac{(-1)^{n-1} \hat{\lambda}^{n-1}}{(n+1) !} e^{n}$,

which, with use of (4.17), reduces to

$\sigma_{R} / \sigma=-2 \sum_{n=2}^{\infty}(-1)^{n-1} \frac{(\hat{\lambda} e)^{n-1}}{(n+1) !}=-2 \sum_{m=1}^{\infty}(-1)^{m} \frac{(\hat{\lambda} e)^{m}}{(m+2) !}>0$,

where the positive non-dimensional parameter $\hat{\lambda}$ is defined in (5.6).

The standard alternating series test then shows that this is a convergent series, regardless of the value of the finite parameter $\hat{\lambda}$. It can in fact further be shown that this relation between the total stress and its work-recoverable part is equivalent to its closed form counterpart (5.5).

\section{References}

1. Adkins JE (1951) Studies in the theory of large elastic deformations, a thesis presented for the degree of PhD, London University

2. Adkins JE, Rivlin RS (1955) Large elastic deformations of isotropic materials X. Reinforcement by inextensible cords. Philos Trans R Soc Lond A 248:201-223

3. Pipkin AC, Rogers TG (1971) Plane deformations of incompressible fiber-reinforced materials. J Appl Mech Trans ASME 38:634-640

4. Spencer AJM (1972) Deformations of fibre reinforced materials. Clarendon Press, Oxford

5. Rogers TG, O’Neil JM (1991) Theoretical analysis of forming flows of fibre-reinforced composites. Comp Manuf 2:153-160

6. Spencer AJM, Soldatos KP (2007) Finite deformations of fibre-reinforced elastic solids with fibre-bending stiffness. Int J Non-linear Mech 42:35-368

7. Soldatos KP (2010) Second-gradient deformations of ideal fibre-reinforced materials II: forming flows of fibre-resin systems when fibres resist bending. J Eng Math 68:179-196

8. Steigmann DJ (2018) Continuum theory of elastic sheets formed by inextensible elasticae. Int J Non-lin Mech 106:324-329 
9. Soldatos KP, Aydogdu M, Gul U (2019) Plane strain polar elasticity of fibre reinforced functionally graded materials and structures. J Mech Mater Struct 14:497-535

10. Soldatos KP, Shariff MHBM, Merodio J (2020) On the constitution of polar fiber-reinforced materials. Mech Adv Mater Struct. https://doi.org/10.1080/15376494.2020.1729449

11. Soldatos KP (2020) On the characterisation of fibrous composites when fibres resist bending - part III: the spherical part of the couple-stress. Int J Solids Struct 202:217-225

12. Holzapfel GA, Gasser TC, Ogden RW (2000) A new constitutive framework for arterial wall mechanics and a comparative study of material models. J Elast 61:1-48

13. Gasser TC, Ogden RW, Holzapfel GA (2006) Hyperelastic modelling of arterial layers with distributed collagen fibre orientations. J R Soc Interface 3:15-35

14. Holzapfel GA, Ogden RW (2015) On the tension-compression switch in soft fibrous solids. Eur J Mech A/Solids 49:561-569

15. Li K, Ogden RW, Holzapfel GA (2018) Modeling fibrous biological tissues with a general invariant that excludes compressed fibers. J Mech Phys Solids 110:38-53

16. Holzapfel GA, Ogden RW, Sherifova S (2019) On fibre dispersion modelling of soft biological tissues: a review. Proc R Soc A 475:2018073

17. Kumar S, Maxwell IZ, Heisterkamp A, Polte TR, Lele TP, Salanga M, Mazur E, Ingber DE (2006) Viscoelastic retraction of single living stress fibers and its impact on cell shape, cytoskeletal organization, and extracellular matrix mechanics. Biophys $\mathbf{J}$ 90:3762-3773

18. Saito T, Kimura S, Nishiyama Y, Akira A (2007) Cellulose nanofibers prepared by TEMPO-mediated oxidation of native cellulose. Biomacromol 8:2485-2491

19. Gennison J-L, Deffieux T, Macé E, Montaldo G, Fink M, Tanter M (2010) Viscoelastic and anisotropic material properties of in vivo muscle tissue assessed by supersonic shear imaging. Ultrasound Med Biol 36:789-801

20. Sokolnikoff IS (1956) Mathematical theory of elasticity. McGraw Hill, New York, NY

21. Soldatos KP (2020) The generalised viscoelastic spring. Proc R Soc A 476:2019088

22. Spencer AJM (1980) Continuum mechanics. Longman, New York

23. Christensen RM (1971) Theory of viscoelasticity: an introduction. Academic Press, New York

24. Tanner RI, Walters K (1998) Rheology: an historical perspective. Elsevier, Amsterdam

25. Mainardi F (2010) Fractional calculus and waves in linear viscoelasticity: an introduction to mathematical models. Imperial College Press, London

26. Knops RJ, Quintanilla R (2021) On the quasi-static approximation in the initial boundary value problem of linearised elastodynamics. J Eng Math 126:11. https://doi.org/10.1007/s10665-020-10072-5

27. Mullins L (1969) Softening of rubber be deformation. Rubber Chem Tech 42:339-362

28. Ogden RW, Roxburgh DG (1999) A pseudo-elastic model for the Mullins effect in filled rubber. Proc R Soc Lond A 455:2861-2877

29. Dorfmann A, Ogden RW (2004) A constitutive model for the Mullins effect with permanent set in particle-reinforced rubber. Int $\mathbf{J}$ Solids Struct 41:1855-1878

30. Soldatos KP (2015) Modelling framework of mass growth II: the general case. Mech Res Commun 65:35-57

31. Soldatos KP (2015) Modelling framework of mass growth III: isochoric growth. Mech Res Comm 70:63-71

32. Soldatos KP (2018) On the dilatation of a compressible Rivlin cube beyond its elastic limit. Int J Non-Lin Mech 106:310-323

Publisher's Note Springer Nature remains neutral with regard to jurisdictional claims in published maps and institutional affiliations. 